\title{
AIRCRAFT ENGINE NOISE SCATTERING BY FUSELAGE AND WINGS - A COMPUTATIONAL APPROACH
}

\author{
D. Stanescu* ${ }^{*}$ M.Y. Hussaini* and F. Farassat ${ }^{\dagger}$ \\ * School of Computational Science and Information Technology, Florida \\ State University, Tallahassee, Florida \\ † NASA Langley Research Center, Hampton, Virginia \\ Proposed running head: Aircraft noise scattering
}

\author{
Correspondence to: \\ Dan Stanescu \\ c/o Susan Kane \\ CSIT/FSU \\ 400 DSL Bldg. \\ Tallahassee, Florida, 32306-4120 \\ Tel.: (850) 644-4753 \\ FAX.: (850) 644-0098 \\ E-mail : dans@csit.fsu.edu
}

Total pages (including cover): 27

Total figures: 12 


\section{List of Figures}

1 A $\zeta=$ const plane through the master element, showing the position of the Gauss-Legendre points inside the element and the position of the flux points on the element faces. . . . . . 16

2 Actual wall-clock time parallel performance for two test cases on up to 16 processors: ideal (full line), 17370-element grid on Origin2000 $(\times)$, 884-element grid on SP3 $(+) \ldots \ldots . . .17$

3 SPL contours for radiation of spinning mode $(2,1)$ at $\omega=$ 8 from an axisymmetric bell-mouth nacelle alone. Nacelle surface and plane $z=0$ through its axis shown. Contour increment $\Delta=2 \ldots \ldots \ldots \ldots \ldots$

4 SPL contours for radiation of spinning mode $(2,1)$ at $\omega=8$ from an axisymmetric bell-mouth nacelle alone. Plane $z=-4$ shown. Trace of mesh decomposition shown on nacelle surface. 19

5 SPL contours for the radiation of spinning mode $(2,1)$ at $\omega=$ 8 in the fuselage-pylon-nacelle configuration in plane $z=-4$. Trace of mesh decomposition shown on the solid surfaces. Contour increment $\Delta=4 \ldots \ldots \ldots \ldots$

6 SPL contours for the radiation of spinning mode $(2,1)$ at $\omega=$ 8 in the complete configuration with wing. Solid surfaces shown only. Contour increment $\Delta=4 \ldots \ldots . . . . .21$

$7 \quad$ SPL contours for the radiation of spinning mode $(2,1)$ at $\omega=$ 8 in the complete configuration with wing. Plane $y=0$ shown only. . . . . . . . . . . . . . . . . . 22

8 SPL contours for the radiation of spinning mode $(2,1)$ at $\omega=$ 8 in the complete configuration with wing. Plane $z=0$ and trace of mesh decomposition on solid surfaces shown. . . . . 23

9 SPL contours for the radiation of spinning mode $(2,1)$ at $\omega=$ 8 in the complete configuration with wing. Plane $z=-4$ and trace of mesh decomposition on solid surfaces shown. . . . . . 24

10 Directivity in the plane $y=0$ for the complete configuration with wing (dashed line) compared with the nacelle alone case (full line). . . . . . . . . . . . . . . . 25

11 SPL $\left(d B\right.$ re. $\left.2 \times 10^{-5} \mathrm{~N} / \mathrm{m}^{2}\right)$ variation on the line $y=z=0$ for the three configurations: nacelle (full line), fuselage $(+)$, and wing $(\times) \ldots \ldots \ldots \ldots \ldots \ldots$

12 SPL $\left(d B\right.$ re. $\left.2 \times 10^{-5} \mathrm{~N} / \mathrm{m}^{2}\right)$ variation on the line $y=0, z=$ -4 for the three configurations: nacelle (full line), fuselage $(+)$, and wing $(\times) \ldots \ldots \ldots \ldots . \ldots \ldots$ 


\section{ABSTRACT}

The paper presents a time-domain method for computation of sound radiation from aircraft engine sources to the far-field. The effects of nonuniform flow around the aircraft and scattering of sound by fuselage and wings are accounted for in the formulation. The approach is based on the discretization of the inviscid flow equations through a collocation form of the Discontinuous Galerkin spectral element method. An isoparametric representation of the underlying geometry is used in order to take full advantage of the spectral accuracy of the method. Large-scale computations are made possible by a parallel implementation based on message passing. Results obtained for radiation from an axisymmetric nacelle alone are compared with those obtained when the same nacelle is installed in a generic configuration, with and without a wing. 


\section{INTRODUCTION}

One of the main sources of aircraft noise is the engine. Most of the measurements and modeling regarding engine noise have been done for an isolated engine $[1,2]$. The far field engine noise of an aircraft is, however, influenced both by the flow around the wings and fuselage and by scattering of sound from aircraft surfaces. Ideally, one would like to be able to compute the radiated noise field that takes into account these effects. One of the motivations behind this is to explore the possibility of reducing the aircraft noise foot-

print by taking advantage of engine and wing location and by manipulating the nonuniform mean flow around the fuselage. This paper presents a spectral method suitable for large-scale simulations of engine noise propagation in a realistic configuration and flow environment around an aircraft.

Recent advances both in computer architecture and computing methods have already prompted researchers to address the fan tone noise problem using Computational Aeroacoustics (CAA) time-domain techniques [3, 4, 5]. A treatment of the acoustic liner boundary condition, which is not obvious in this context since the liner impedance data is usually available in the frequency domain, was recently proposed by Ozyoruk and Long [6, 7]. Stanescu et al. [5] used a multi-domain spectral method for fan tone noise radiation and showed that accurate results can be obtained with a relatively small (between four and five, depending on the highest polynomial degree) number of points per wavelength. The spectral multi-domain method is geometrically flexible, as it only requires finite-element type grids that can be generated for usual aircraft configurations with commercially available packages. Although this fact makes practical three-dimensional problems tractable, the computer resources needed for frequencies typical of those encountered in fan noise are still very large. Such problems can only be solved if a proper parallelization strategy is used in order to make best use of the modern distributed memory computers available.

The discontinuous Galerkin (DG) method for hyperbolic systems of conservation laws has been studied by Cockburn and Shu [8] in detail. A spectral element implementation was proposed by Kopriva et al. [9], and several studies have been performed on its dissipation and dispersion properties $[10,11,12]$. These studies demonstrate clearly its advantages in terms of accuracy when applied to wave propagation problems. The DG method is otherwise completely similar to the multi-domain spectral method [5] both in formulation and implementation, and has therefore been considered as the discretization technique for this work. Through a proper choice of the flux computation points, the method only requires communication between 
elements that have common faces, and is therefore very well suited for parallelization using message passing. In this setting, an initial ordering of the elements pertaining to each processor allows communication of data for elements on partition boundaries to be overlapped with computation of the fluxes for those elements inside each partition, thus minimizing the impact of communication on the overall performance.

The present paper is organized as follows. The next section briefly describes the DG method and its numerical implementation. Issues related to geometry representation, grid generation and parallelization strategy are all addressed. The numerical results section then presents computations of the sound field generated by a single spinning mode propagating around an axisymmetric nacelle, and compares the results with those obtained for propagation of the same mode when the nacelle is mounted in a generic fuselage-pylon-nacelle configuration, both with and without a wing. The conclusions section ends the paper.

\section{THE DG SPECTRAL ELEMENT METHOD}

\subsection{GOVERNING EQUATIONS}

The three-dimensional, nonlinear Euler equations of gas dynamics in conservation form,

$$
\frac{\partial \tilde{Q}}{\partial t}+\sum_{d=1}^{3} \frac{\partial \tilde{F}_{d}}{\partial x_{d}}=0
$$

are considered here to model the noise propagation process. The components of the state and flux vector are:

$$
\tilde{Q}=\left(\begin{array}{c}
\rho \\
\rho v_{1} \\
\rho v_{2} \\
\rho v_{3} \\
\rho E
\end{array}\right), \tilde{F}_{d}=\left(\begin{array}{c}
\rho v_{d} \\
\rho v_{1} v_{d}+p \delta_{1 d} \\
\rho v_{2} v_{d}+p \delta_{2 d} \\
\rho v_{3} v_{d}+p \delta_{3 d} \\
(\rho E+p) v_{d}
\end{array}\right)
$$

where $\rho$ is the fluid density, $v_{i}, i=1,2,3$ the fluid velocity components in the three Cartesian directions, $p$ the pressure, $E$ the total internal energy per unit mass, and $\delta_{i j}$ the Kronecker delta symbol. 


\subsection{DG DISCRETIZATION}

The computational domain is divided into non-overlapping general hexahedral elements that can have curved boundaries. Each element is then individually mapped onto the master element $\Omega_{M}=[-1,1]^{3}$ using an isoparametric transformation. Under the mapping, Eq. (1) becomes

$$
\frac{\partial Q}{\partial t}+\sum_{d=1}^{3} \frac{\partial F_{d}}{\partial \xi_{d}}=0
$$

where the new variables are the transformed components of the state and flux vector

$$
Q=J \tilde{Q}, \quad F_{d}=J \sum_{m=1}^{3} \frac{\partial \xi_{d}}{\partial x_{m}} \tilde{F}_{m}
$$

and $J$ is the Jacobian of the transformation

$$
J=\operatorname{det}\left(\frac{\partial\left(x_{1}, \ldots, x_{3}\right)}{\partial\left(\xi_{1}, \ldots, \xi_{3}\right)}\right)
$$

The computational space coordinates will be denoted here by both $\left(\xi_{1}, \xi_{2}, \xi_{3}\right)$ and $(\xi, \eta, \zeta)$ for convenience.

Let the space of polynomials of degree $N$ in $\xi \in[-1,1]$ be denoted by $P_{N}$. A basis for this space can be constructed using the Lagrange interpolating polynomials

$$
h_{i}(\xi)=\prod_{j=0, j \neq i}^{N} \frac{\left(\xi-\xi_{j}\right)}{\left(\xi_{i}-\xi_{j}\right)}, \quad h_{i}\left(\xi_{j}\right)=\delta_{i j}
$$

through the $N+1$ Gauss-Legendre [13] quadrature nodes $\xi_{i}, i=0,1, \ldots, N$. The three-dimensional solution $Q$ is searched in a trial space obtained as the tensor product of one-dimensional degree $N$ polynomials for each coordinate direction, $P_{N}^{3}=P_{N}(\xi) \times P_{N}(\eta) \times P_{N}(\zeta)$, hence it takes the form

$$
Q(t, \xi, \eta, \zeta)=\sum_{i=0}^{N} \sum_{j=0}^{N} \sum_{k=0}^{N} Q_{i j k}(t) h_{i}(\xi) h_{j} \eta h_{k} \zeta
$$


where $Q_{i j k}(t)$ denote pointwise values of $Q$ at time $t$. In view of a Galerkin discretization, the residual is required to be orthogonal to the same trial space within each element. Thus, the DG spectral element method looks for a solution of the system

$$
\left(Q_{t}, \phi_{i j k}\right)+\left(\nabla_{\xi} \cdot F, \phi_{i j k}\right)=0
$$

to be satisfied for all $i, j, k=0,1, \ldots, N$. Here $(\cdot, \cdot)$ represents the usual $L^{2}$ inner product, and $\phi_{i j k}=h_{i}(\xi) h_{j}(\eta) h_{k}(\zeta)$ are the basis functions of $P_{N}^{3}$. Using the divergence theorem, the equation is recast as

$$
\left(Q_{t}, \phi_{i j k}\right)+\int_{\partial \Omega_{M}} \phi_{i j k} F \cdot n d S=\left(F, \nabla_{\xi} \phi_{i j k}\right)
$$

In order to solve these equations efficiently, the integrals are computed using Gauss quadrature

$$
\int_{-1}^{1} f(\xi) d \xi=\sum_{m=0}^{N} f\left(\xi_{m}\right) w_{m}, \forall f \in P_{2 N+1}
$$

$w_{m}$ being the Gauss weights. This replacement is exact for elements with straight edges, in which case $J$ is a polynomial of degree one. In the general case of elements with curved boundaries, a discretization error is incurred in the use of the numerical quadrature rule Eq. (10).

Expanding the boundary integral and performing some algebraic manipulation, the final discrete form of the equations governing each variable at the Legendre-Gauss points can be found to be

$$
\frac{d Q_{i j k}}{d t}=-\left[D^{\xi}+D^{\eta}+D^{\zeta}\right] F
$$

where the right-hand side is a sum of discrete differential operators acting on the flux values of an element, which include values on the element faces. Defining the contribution due to the volume term on the right-hand side of 
Eq. (9) as

$$
d^{\xi} F=\sum_{m=0}^{N} F_{1}^{m j k}\left(h_{i}^{\prime}, h_{m}\right)_{N}
$$

where the discrete inner product $(u, v)_{N}=\sum_{n=0}^{N} u_{n} v_{n} w_{n}$ was introduced, the differential operator for $\xi$ for example can be written

$$
D^{\xi} F=\frac{1}{w_{i}}\left[F_{1}^{*}\left(1, \eta_{j}, \zeta_{k}\right) h_{i}(1)-F_{1}^{*}\left(-1, \eta_{j}, \zeta_{k}\right) h_{i}(-1)-d^{\xi} F\right]
$$

and the remaining two follow by obvious permutations. In this equation the notation $F^{*}$ denotes a common face flux as explained below. The position of the Gauss-Legendre points inside the element, as well as the position of the corresponding flux points on the element faces where $F^{*}$ must be evaluated, is shown in Figure 1, drawn for one $\zeta=$ const plane and $N=2$.

It is important to note that the operation in Eq. (12) is actually a onedimensional operation that is expressed in the computer code as the multiplication of a matrix of entries $d_{i m}=\left(h_{i}^{\prime}, h_{m}\right)_{N}$ with a vector of size $N+1$ containing the values of the $F_{1}$ flux component at the Legendre-Gauss points on a line $i=$ const inside the element. These flux values can be computed directly from the values of the state vector which are readily available. The values of the state vector at the face points of each element, i.e. $Q\left(1, \eta_{j}, \zeta_{k}\right)$ and $Q\left(-1, \eta_{j}, \zeta_{k}\right)$ for the $\xi=$ const faces, are obtained by evaluating the interpolating polynomial based on the Gauss-Legendre points at $\xi= \pm 1$, again a one-dimensional (implemented as a vector dot product) operation. However, these values can not be used directly in computing the pointwise flux values on the element faces, as they are not uniquely defined. Indeed, for each face point, two different values are obtained for the value of the state vector $Q$, one from each of the two neighboring elements. In order to define a unique flux, a Riemann problem [14] is solved at each flux face point with the two different states as initial data, just as in the finite volume method. Letting the superscripts $L$ and $R$ denote the two (left and right) neighboring elements of a face, the application of the Riemann solver can be written

$$
F_{d}^{*}(\cdot)=F_{d}^{*}\left(Q^{R}(\cdot), Q^{L}(\cdot)\right)
$$

and completes the evaluation of the right-hand side of Eq. (11). Upon this, 
it becomes on ordinary differential equation which is integrated in time using a low-storage Runge-Kutta scheme optimized for wave propagation [15].

The computer code thus obtained can be used for computing both a steady mean flow, when appropriate inflow/outflow boundary conditions are specified at the fan face and the far field, and the propagation of acoustic waves superposed on such a mean flow previously obtained. For acoustic computations, once the mean flow is known, the boundary condition on acoustic source surfaces is allowed to become a function of time (corresponding to a source term in the governing equations), and the acoustic pressure is obtained as the difference between the computed value of $p$ and the mean flow pressure. Radiation boundaries are treated by a damping layer approach, similar to Stanescu [5]. The damping layer is activated only for acoustic computations. This precludes the need for a time-dependent radiation boundary condition, i.e. the boundary condition at the exterior limit of the damping layer is the same inflow/outflow characteristic boundary condition that can be used to compute the steady mean flow field.

\subsection{ISOPARAMETRIC ELEMENTS}

To obtain an accurate representation of the underlying geometry, the ICEMCFD Hexa [16] commercial package has been used for hexagonal grid generation around the configurations of interest. Once an unstructured grid of hexahedra was obtained, a new feature of this package allows for the generation of points along each of the edges of the hexahedral mesh according to a prescribed distribution, which can be either a Legendre-Lobatto or a Chebyshev-Lobatto distribution in arclength, for a specified polynomial degree $N$. The resulting points are written to a data file which can be read in a computer code and used to define the geometry with the same accuracy as the solution. All the necessary point coordinates can then be computed by interpolation based on the spectral interpolants along the edges (to obtain coordinates at the Gauss points from the Lobatto points on the edges) followed by three-dimensional transfinite interpolation [17] on the faces and inside the elements. The intermediary use of the Lobatto points is found necessary because they include the eight corners of the hexahedra (and hence the end points for each edge of the grid) which can thus be guaranteed to have a unique position. This would not be the case if their coordinates were created by interpolation from data at Gauss points (they would be different due to interpolation and truncation errors). 


\subsection{PARALLEL IMPLEMENTATION}

The DG spectral element method is particularly well suited for a parallel implementation through message passing as the computation is naturally structured by elements, with most of the work done inside the element i.e. evaluation of derivatives in Eq. (11). Here a brief description of the parallel implementation is given. The program starts with a preprocessing step wherein the underlying hexahedral mesh is decomposed in as many partitions as the number of processors using the multilevel graph partitioning techniques in METIS [18]. Each processor creates the connectivity for its grid partition, which includes a list of elements ("cut elements" henceforth) having one or more "cut faces" in common with elements in neighboring partitions.

One stage of the Runge-Kutta time integration scheme for the parallel DG algorithm can then be described as follows:

- Compute state vector values at the face flux points through interpolation for all cut elements.

- Post non-blocking send/receive for the state vector values on the cut faces. Each processor sends the values interpolated from the cut elements it owns to the corresponding neighboring partition.

- Compute state vector values at the face flux points through interpolation in the regular elements inside each partition.

- Compute unique fluxes through the Riemann solver for regular faces, Eq. (14).

- Test for completed send/receive.

- Compute unique face fluxes through the Riemann solver for cut faces, Eq. (14).

- Compute residual and update the state vector according to Eq. (11).

The parallel performance of this algorithm has been tested on several architectures, for various grid sizes and polynomial degrees $N$. Results obtained for two grid sizes are presented here. The first one is a small grid of 884 elements with polynomial degree $N=12$, which has been run on an IBM SP3 machine with $375-\mathrm{MHz}$ Power3 processors, each with $512 \mathrm{MB}$ of memory. The second one is a medium-size (17370 elements) grid used for the fuselage-nacelle configuration, run with $N=4$ on an SGI Origin2000 
machine with $250 \mathrm{MHz}$ R10000 processors. Both cases could be run on a single processor, and Figure 2 shows the performance on up to 16 processors as obtained by comparing the wall-clock time with the single processor run.

\section{NUMERICAL EXAMPLES}

The methodology described above has been validated by comparison with both analytical solutions and experiments in [5]. The computations presented below have been performed in order to study the feasibility of the method for large scale computations, its parallel performance, the eventual problems raised by such computations, as well as to gain some initial physical insight into the influence of the fuselage and/or other surfaces on the sound field of a nacelle. Only inlet noise radiation of a single mode is computed in the cases presented here. The incoming pressure perturbation is specified as a circular duct eigensolution [19],

$$
p^{\prime}(x, r, \theta, t)=A_{m \mu} J_{m}\left(k_{m \mu} r\right) \cos \left(k_{x} x+m \theta+\phi_{m \mu}-\omega t\right)
$$

where $m$ is the azimuthal order of the spinning mode, $J_{m}$ the Bessel function of the first kind, $\phi_{m \mu}$ is the phase and $A_{m \mu}$ the modal amplitude. The density and velocity perturbations are obtained from the equations of motion. To keep the paper at a reasonable length, results are presented only for a stationary medium. Mean flow effects will be discussed in a subsequent work.

Remarks on non-dimensionalization. In all cases, lengths were nondimensionalized using the radius of the inlet duct as reference value. The same amplitude of the incoming acoustic wave was used for all computations. The pressure amplitude of the incoming acoustic modes at the fan face was set to $A_{m \mu}=141.6 \mathrm{~N} / \mathrm{m}^{2}$, and the sound pressure level (SPL) is reported in decibels relative to the usual reference pressure $p_{\text {ref }}=2 \times 10^{-5} \mathrm{~N} / \mathrm{m}^{2}$.

\subsection{NACELLE ALONE}

The first computation was performed for the sound field radiated from an axisymmetric bell-mouth nacelle. The radius of the leading edge of the bell-mouth has been chosen to be $1 / 4$ of the radius of the inlet duct, and no center-body (hub) was added. Radiation of the $(2,1)$ spinning mode 
at reduced frequency $\omega=8.0$ in a quiescent fluid has been modeled on a grid made of $E=9919$ elements. The polynomial degree used was $N=6$, leading to an average of about five points per wavelength (largest element edge length is 1.06). There are a total of 3,402,217 Gauss-Legendre points in the domain. The nacelle casing extends on the $x$-axis (the symmetry axis) from $x=-1.25$ to $x=3.0$, and the domain extends from $x=-15$ to $x=8$, with a damping layer width of 3 in each direction. Incoming acoustic waves are specified on the source surface which is the circular disk with center at $(0,0,0)$ and unitary radius.

Figure 3 shows the SPL contours on the surface of the nacelle and in a plane that cuts the nacelle through its symmetry axis. Since no scattering surfaces other than the engine exist, the radiation is symmetric with respect to the engine axis. Figure 4 shows the contours in the plane $z=-4$. This computation was also used as an initial test of the radiation boundary conditions, which perform quite well in this case (very little waviness of the SPL contours is noticeable towards the interior limits of the damping layer). The main radiation lobe makes an angle of about $\theta=46$ degrees with the $x$-axis, a value significantly different from $\theta=57$ degrees obtained using the analytical formula derived by Rice [20] for infinitely thin cylindrical ducts,

$$
\cos \theta=\sqrt{1-\frac{k_{m \mu}^{2}}{\omega^{2}}}
$$

where $k_{m \mu}=6.70613$ is the circular duct eigenvalue for this mode. This difference is most probably caused by the large thickness of the nacelle lip (half of the inlet duct radius).

\subsection{FUSELAGE-NACELLE CONFIGURATION}

The second case considered a configuration obtained by joining the same nacelle with a cylindrical fuselage through an elliptic cylinder pylon. The cylinder representing the fuselage has a radius of 4.5 and its center is situated at $y=-7$. The grid is made up of $E=17370$ elements of the same order $N=6(5,957,910$ Gauss-Legendre points). The minimum and maximum values of the coordinates are $(-18,-7,-9)$ and $(8,10,8)$ respectively, and the damping layer has the same width as in the previous case, with the difference that the plane $y=-7$ does not have an adjacent damping 
layer in the direction perpendicular on it. Instead, a zero normal acoustic velocity boundary condition is used on this plane to simulate the effect of a symmetric configuration with engines in opposite phase on both parts of the fuselage.

As can be seen in Figure 5, the presence of the fuselage creates a series of secondary radiation lobes due to the reflection of the nacelle lobes from the surface and to the various interferences that occur. The structure of the radiated sound field becomes thus quite complex. The presence and extent of the footprint of the $S P L=0$ contour level in this plane is a measure of the effect of the fuselage in increasing the noise levels compared to the nacelle alone case. The figure also shows the trace of mesh decomposition on the solid surfaces defining the geometry.

\subsection{FUSELAGE-WING-NACELLE CONFIGURATION}

The geometry of the previous case was augmented with a wing of elliptic cross-section, slightly swept and mounted below the nacelle. The center of the ellipse defining the cross-section of the wing is situated in the plane $z=-3$. A grid of $E=22843$ elements was generated in this case (for $N=6$ this corresponds to 7,835,149 discretization points). In all other respects, the definition of the domain is similar to the previous case. Shown in Figure 6 are the SPL contours on the solid surfaces defining the geometry for this case. The presence of the wing is responsible for a complex, considerably asymmetric, sound field due to sound scattering from its leading and trailing edges. A clear picture of the effect of the wing can be obtained from figure 7, which shows the SPL levels in the plane $y=0$. In this plane, the leading and trailing edge of the wing are situated at $x=-7.2$ and $x=-1.5$, respectively. As can be expected, although in the plane $z=0$ (above the wing) the noise levels are, at most locations, higher than in the preceding case, in a plane below the wing the noise levels are remarkably lower, due to the upward reflection of the sound field by the wing surface, the difference immediately below the wing lying in the $10-15 \mathrm{~dB}$ range. The corresponding contour plots are given in figures 8 and 9 . It can be noticed in these figures that the damping layer region is not large enough to eliminate all reflections from the boundaries, as the contours are jagged towards the limits of the domain. The directivity at twelve nacelle radii from the center of the source disc for this case is compared with the nacelle alone data in figure 10. The shielding effect of the wing below the nacelle (negative $\theta$ values) is clearly visible.

For a quantitative comparison of the three cases, SPL data was extracted 
in the field along several lines. Two such cases, representative for the increasing complexity of the sound field, are presented in figures 11 and 12 . The interference patterns and various scattering effects that can be identified from these plots render good predictions based on simple analytical methods extremely difficult, if not impossible.

\section{CONCLUSIONS}

The results presented here show that a more complete and accurate modeling of tone noise radiated from aircraft engines, including the effect of scattering from wing and fuselage, is now possible on the large distributed memory parallel computers available nowadays. This can have major impact in the design stage, in terms of affecting the noise footprint of the aircraft by changing the relative engine/wing positioning and altering the wing planform. The paper discusses a time domain methodology that is being developed to perform such studies. The reduced frequency for which results are presented here is lower than the usual values encountered at approach flight in current turbofans. However, it is our opinion that the parallelization strategy adopted for this method makes the study of the usual range of blade passage frequency values feasible on the supercomputers available nowadays. Further work, including the effect of mean flow and the sound radiated from the bypass exhaust, will be presented in the near future.

\section{Acknowledgements}

The support of the first author by NASA grant NAG-1-01031 is gratefully acknowledged.

\section{References}

[1] A.V. Parrett and W. Eversman 1986 AIAA Journal 24, 753-760. Wave Envelope and Finite Element Approximations for Turbofan Noise Radiation in Flight.

[2] M. Nallasamy 1997 Journal of Aircraft 34, 387-393. Computation of Noise Radiation from Fan Inlet and Aft Ducts. 
[3] Y. Ozyoruk and L.N. Long 1996 AIAA Journal 34, 894-901. Computation of Sound Radiating from Engine Inlets.

[4] C.L. Rumsey, R.T. Biedron, F. Farassat and P.L. Spence 1998 Journal of Sound and Vibration 213, 643-664. Ducted-Fan Engine Acoustic Predictions Using a Navier-Stokes Code.

[5] D. Stanescu, D. Ait-Ali-Yahia, W.G. Habashi and M.P. Robichaud 1999 AIAA Journal 37, 296-302. Multidomain Spectral Computations of Sound Radiation from Ducted Fans.

[6] Y. Ozyoruk and L.N. Long 1997 Journal of Computational Acoustics 5, 277-296. A Time-Domain Implementation of Surface Acoustic Impedance Condition With and Without Flow.

[7] Y. Ozyoruk 2002 AIAA Journal 40, 450-455. Parallel Computation of Forward Radiated Noise of Ducted Fans with Acoustic Treatment.

[8] B. Cockburn and C.-W. Shu 1989 Journal of Computational Physics 84, 90-113. TVB Runge-Kutta local projection discontinuous Galerkin finite element method for conservation laws. III. One dimensional systems.

[9] D.A. Kopriva, S.L. Woodruff and M.Y. Hussaini 2000 in Discontinuous Galerkin Methods, Lecture Notes in Computational Science and Engineering 11 355-361. Berlin: Springer-Verlag. Discontinuous Spectral Element Approximation of Maxwell's Equations.

[10] F.Q. Hu, M.Y. Hussaini and P. Rasetarinera 1999 Journal of Computational Physics 151, 921-946. An Analysis of the Discontinuous Galerkin Method for Wave Propagation Problems.

[11] P. Rasetarinera, M.Y. Hussaini and F.Q. Hu 2000 in Discontinuous Galerkin Methods, Lecture Notes in Computational Science and Engineering 11 407-412. Berlin: Springer-Verlag. Some Remarks on the Accuracy of a Discontinuous Galerkin Method.

[12] D. Stanescu, D.A. Kopriva and M.Y. Hussaini 2000 Journal of Scientific Computing 15, 149-171. Dispersion Analysis for Discontinuous Spectral Element Methods.

[13] C. Canuto, M.Y. Hussaini, A. Quarteroni and T.A. Zang 1988 Spectral Methods in Fluid Dynamics. Berlin: Springer-Verlag, second edition. 
[14] P.L. Roe 1981 Journal of Computational Physics 43, 357-372. Approximate Riemann Solvers, Parameter Vectors, and Difference Schemes.

[15] D. Stanescu and W.G. Habashi 1998 Journal of Computational Physics 143, 674-681. 2N-Storage Low Dissipation and Dispersion Runge-Kutta Schemes for Computational Acoustics.

[16] ICEMCFD Online Tutorials and Training Manual. Available on WWW at URL http://www.icemcfd.com.

[17] W.J. Gordon and C.A. Hall 1973 International Journal for Numerical Methods in Engineering 7, 461-477. Construction of curvilinear co-ordinate systems and applications to mesh generation.

[18] G. Karypis and V. Kumar. METIS: A Software Package for Partitioning Unstructured Graphs, Partitioning Meshes, and Computing FillReducing Orderings of Sparse Matrices. Available on WWW at URL http://www.cs.umn.edu/ karypis.

[19] J.M. Tyler and T.G. Sofrin 1962 SAE Transactions 70, 309-332. Axial Flow Compressor Noise Studies.

[20] E.J. Rice, M.F. Heidmann and T.G. Sofrin 1979 NASA TM-79030 Modal Propagation Angles in a Cylindrical Duct with Flow and their Relation to Sound Radiation. 


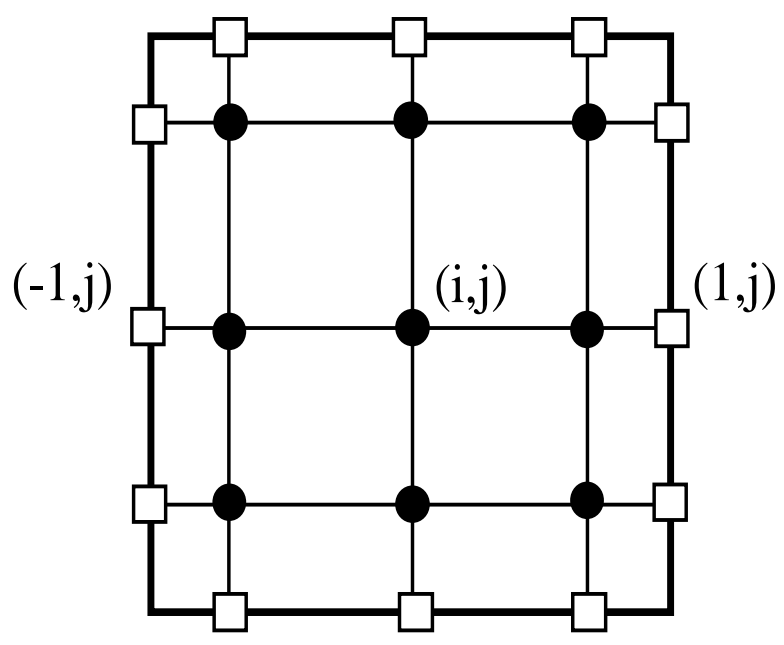

Figure 1: A $\zeta=$ const plane through the master element, showing the position of the Gauss-Legendre points inside the element and the position of the flux points on the element faces. 


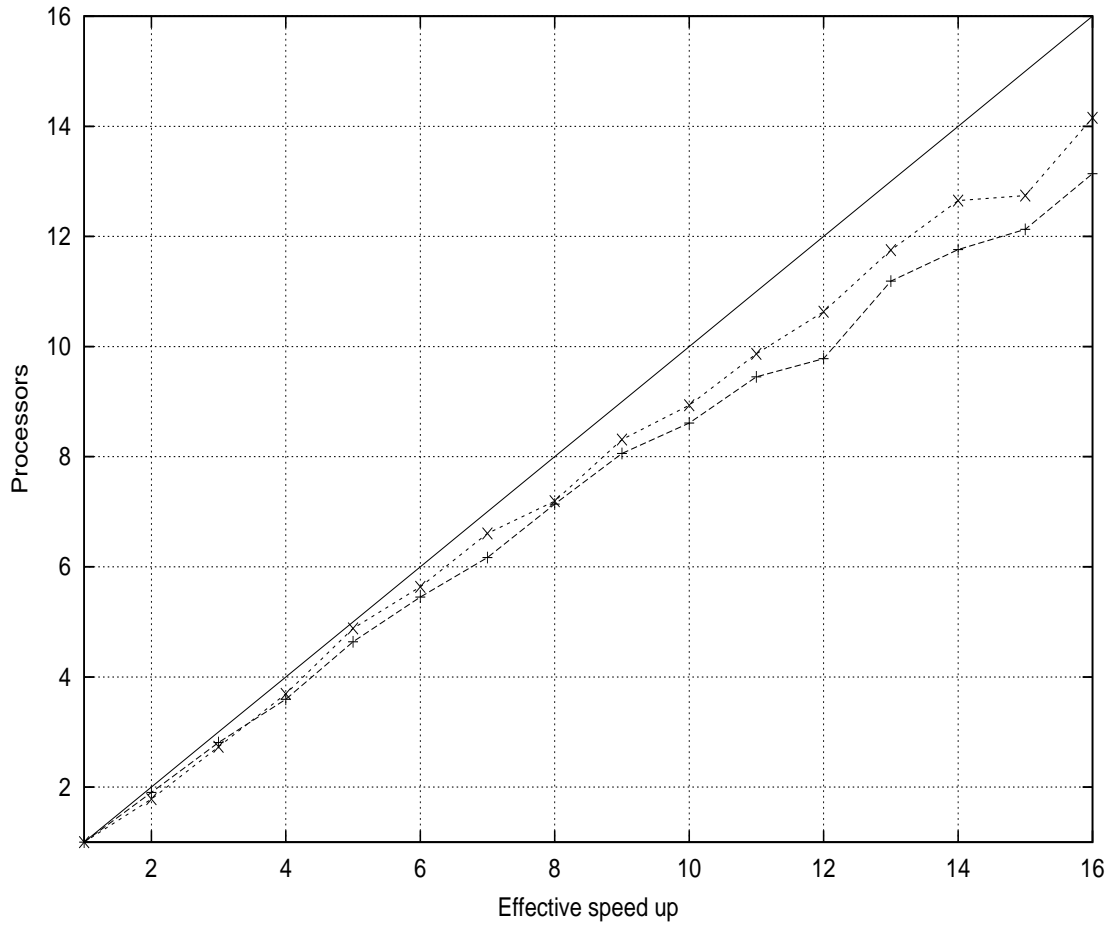

Figure 2: Actual wall-clock time parallel performance for two test cases on up to 16 processors: ideal (full line), 17370-element grid on Origin2000 $(\times)$, 884-element grid on SP3 (+). 


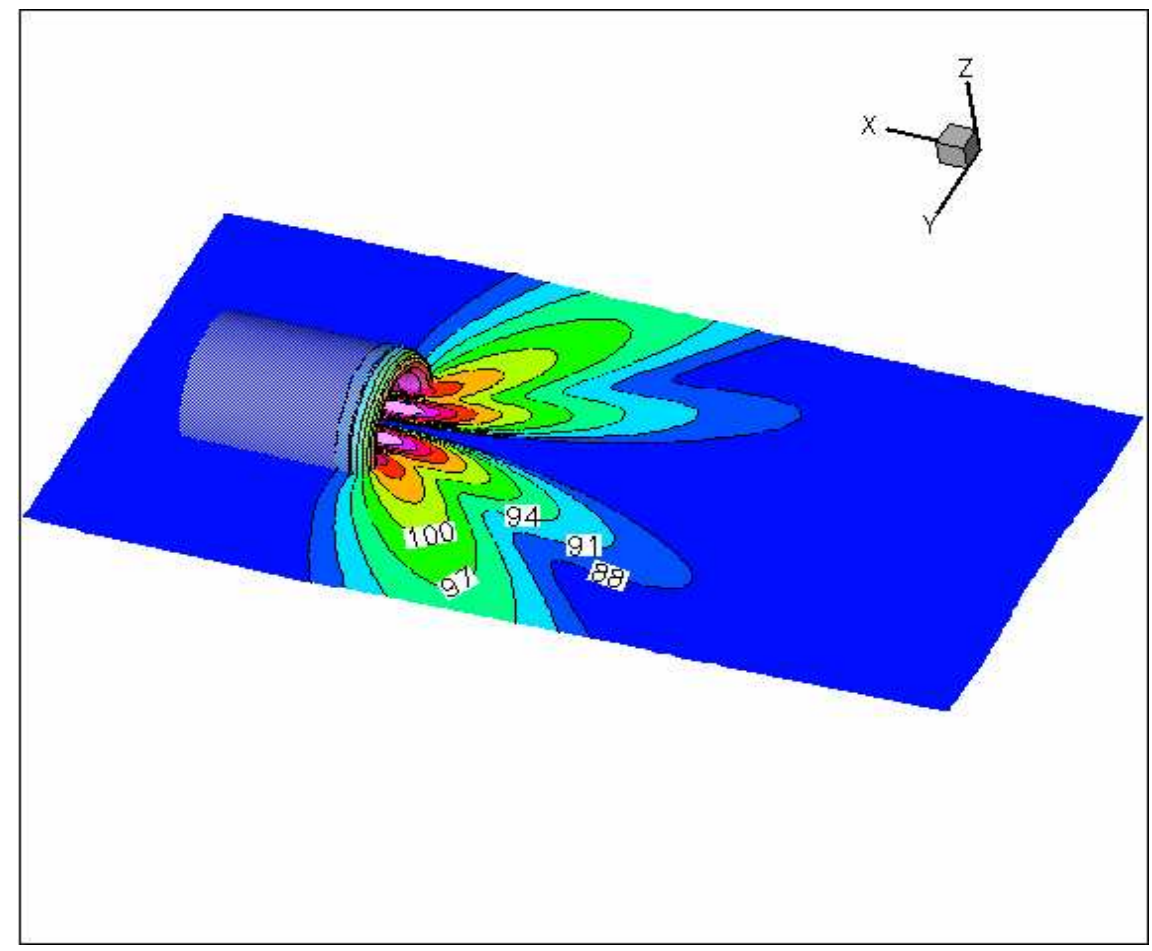

Figure 3: SPL contours for radiation of spinning mode $(2,1)$ at $\omega=8$ from an axisymmetric bell-mouth nacelle alone. Nacelle surface and plane $z=0$ through its axis shown. Contour increment $\Delta=2$. 


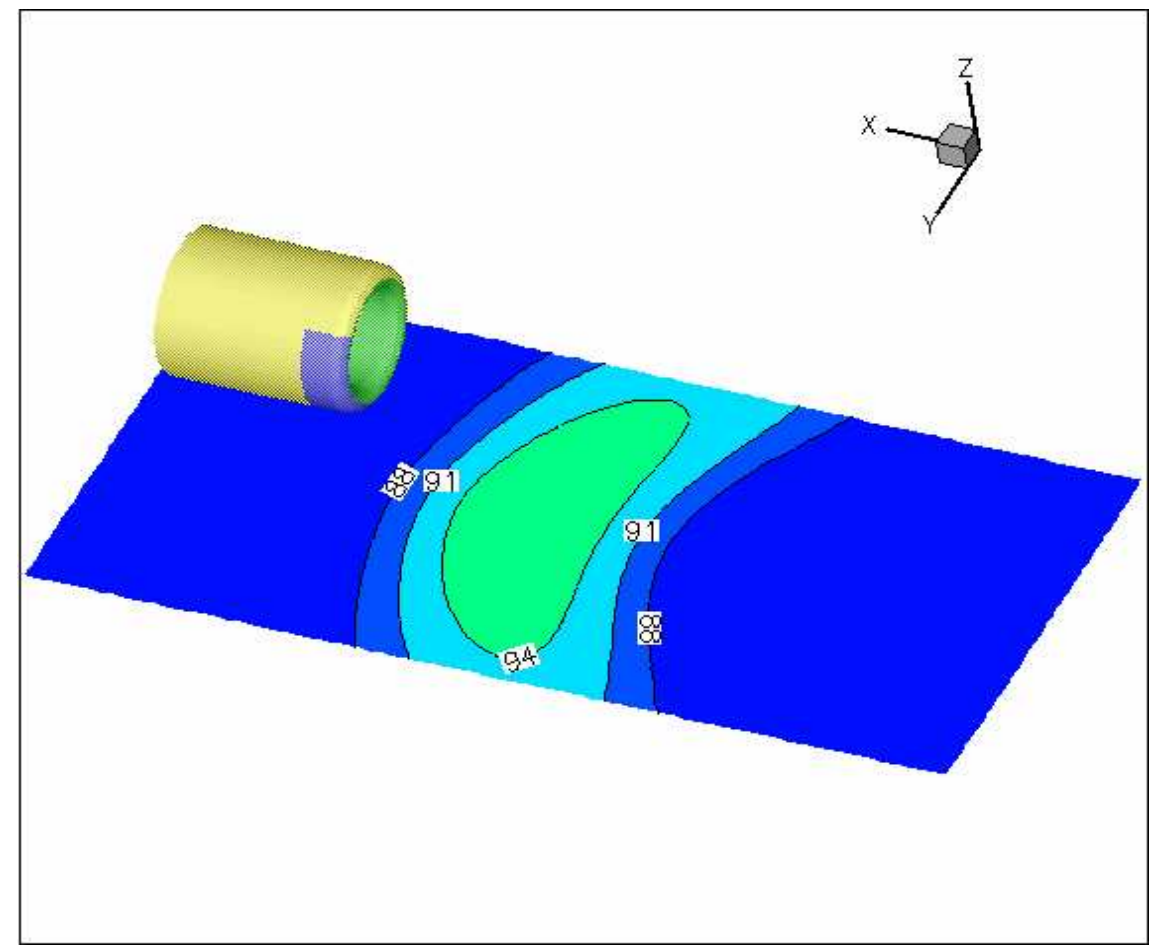

Figure 4: SPL contours for radiation of spinning mode $(2,1)$ at $\omega=8$ from an axisymmetric bell-mouth nacelle alone. Plane $z=-4$ shown. Trace of mesh decomposition shown on nacelle surface. 


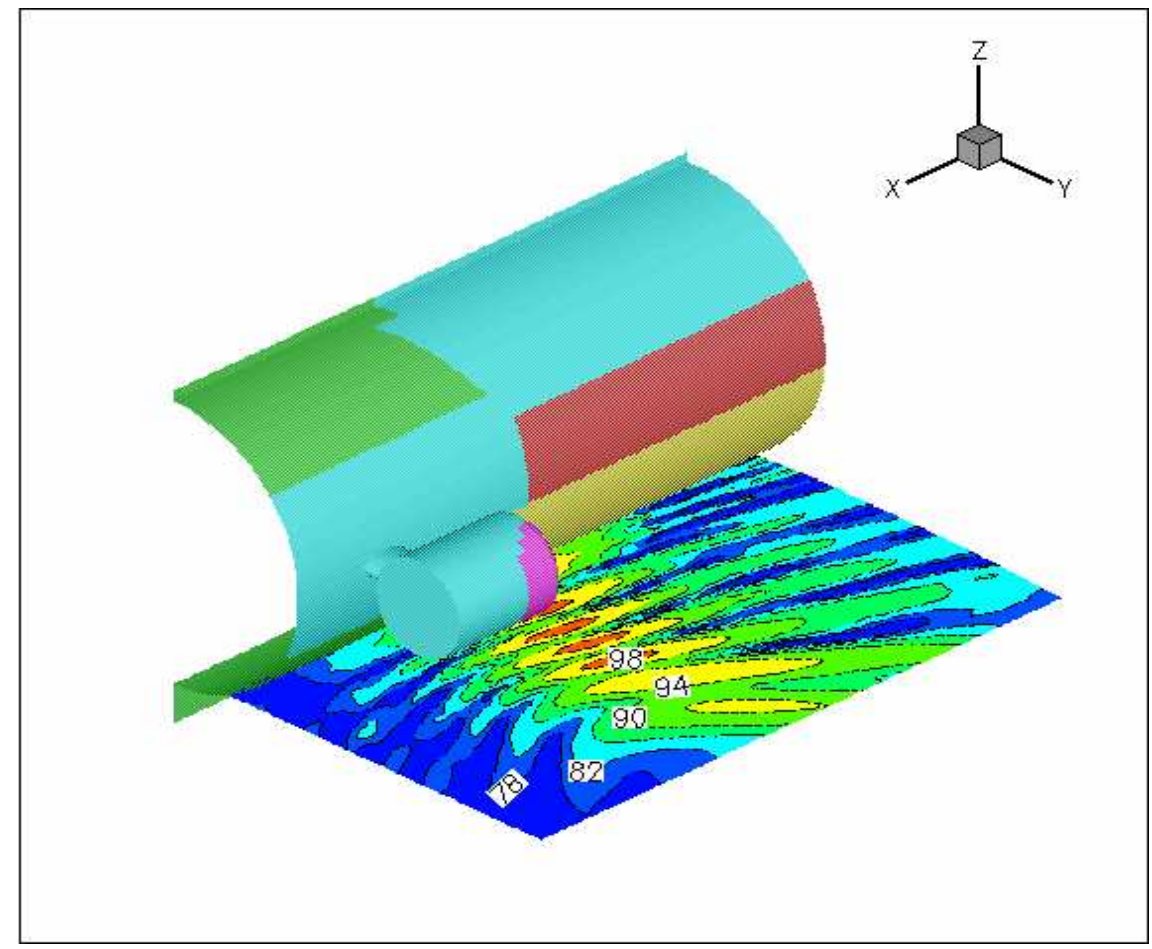

Figure 5: SPL contours for the radiation of spinning mode $(2,1)$ at $\omega=8$ in the fuselage-pylon-nacelle configuration in plane $z=-4$. Trace of mesh decomposition shown on the solid surfaces. Contour increment $\Delta=4$. 


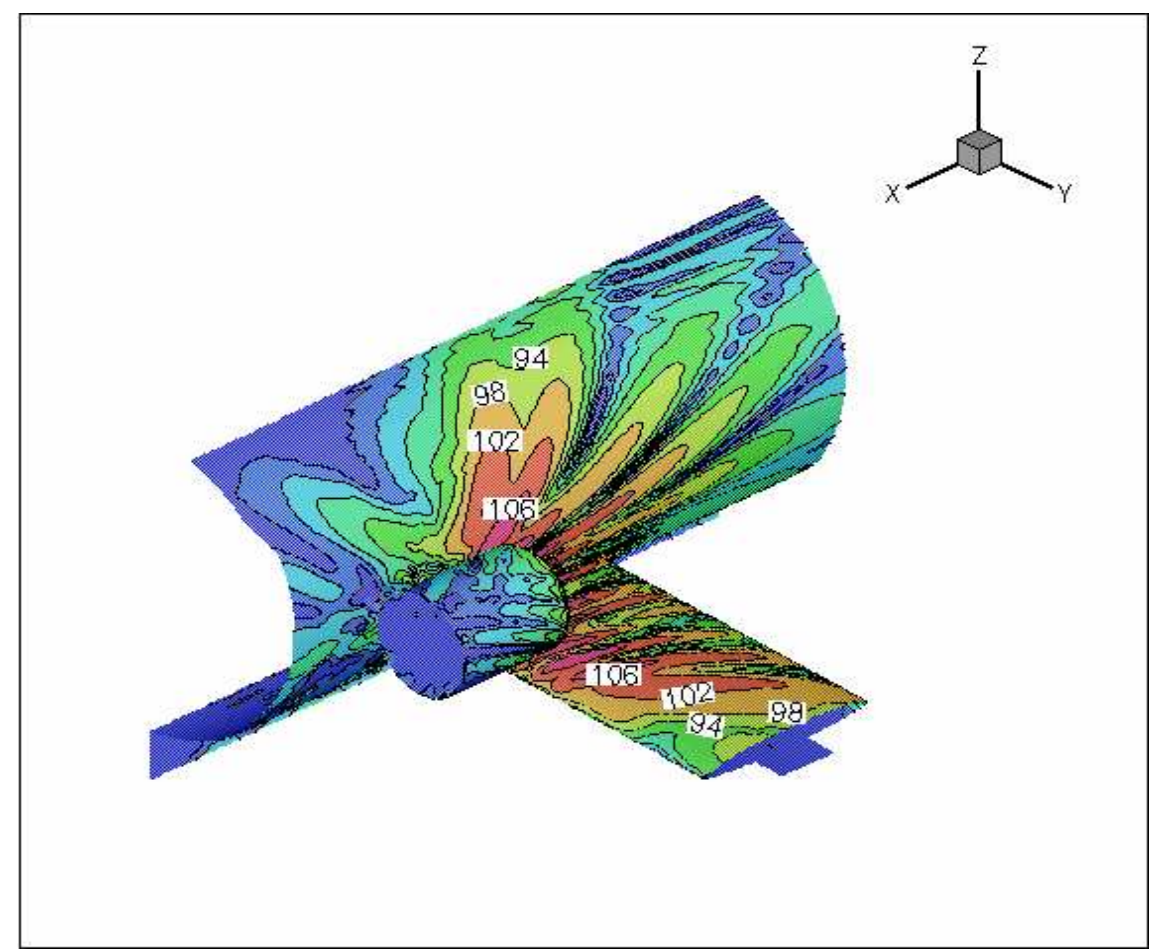

Figure 6: SPL contours for the radiation of spinning mode $(2,1)$ at $\omega=8$ in the complete configuration with wing. Solid surfaces shown only. Contour increment $\Delta=4$. 


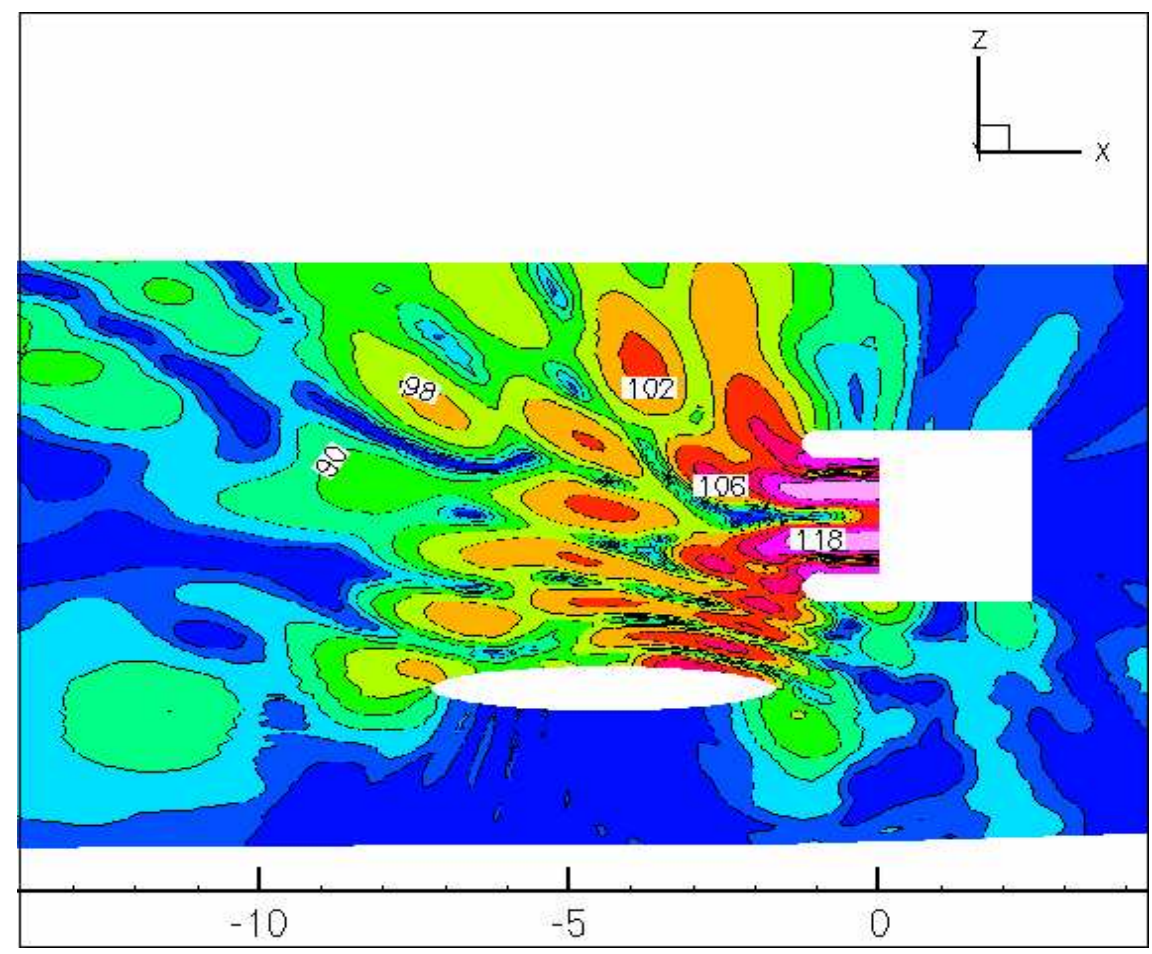

Figure 7: SPL contours for the radiation of spinning mode $(2,1)$ at $\omega=8$ in the complete configuration with wing. Plane $y=0$ shown only. 


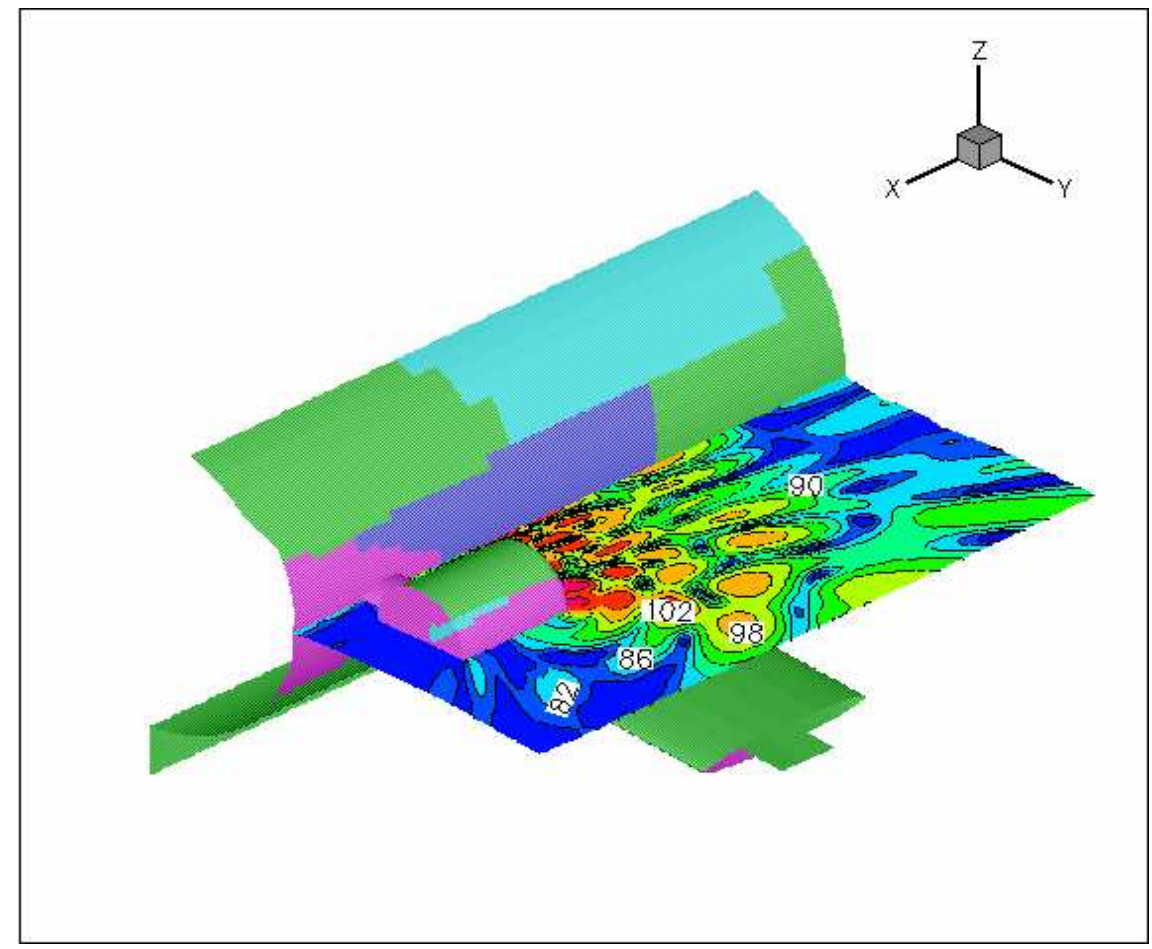

Figure 8: SPL contours for the radiation of spinning mode $(2,1)$ at $\omega=8$ in the complete configuration with wing. Plane $z=0$ and trace of mesh decomposition on solid surfaces shown. 


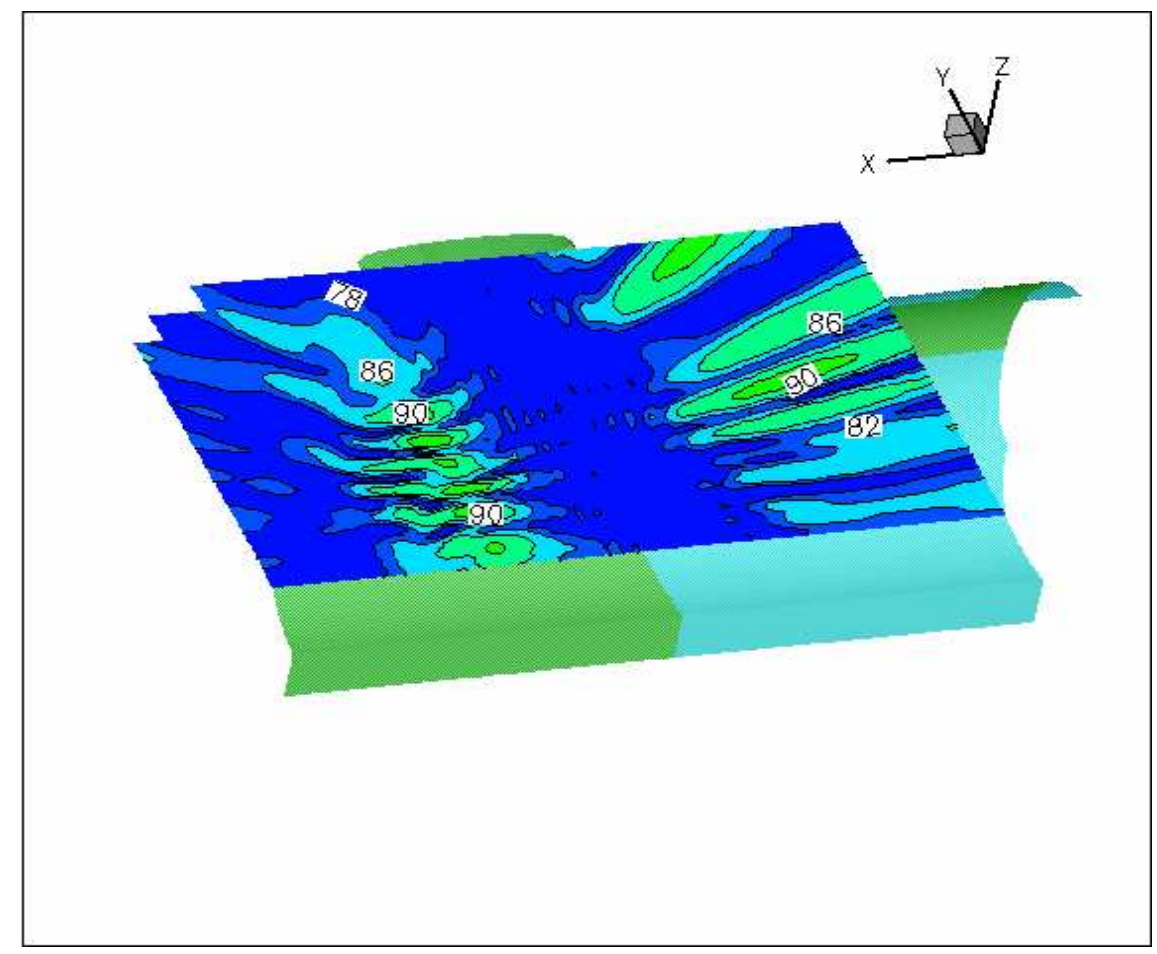

Figure 9: SPL contours for the radiation of spinning mode $(2,1)$ at $\omega=8$ in the complete configuration with wing. Plane $z=-4$ and trace of mesh decomposition on solid surfaces shown. 


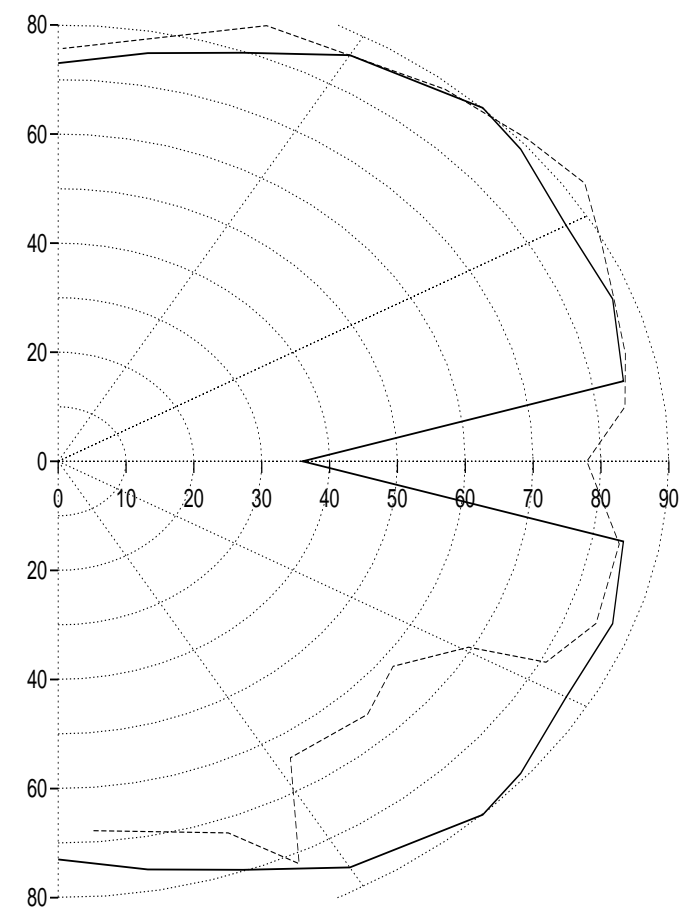

Figure 10: Directivity in the plane $y=0$ for the complete configuration with wing (dashed line) compared with the nacelle alone case (full line). 


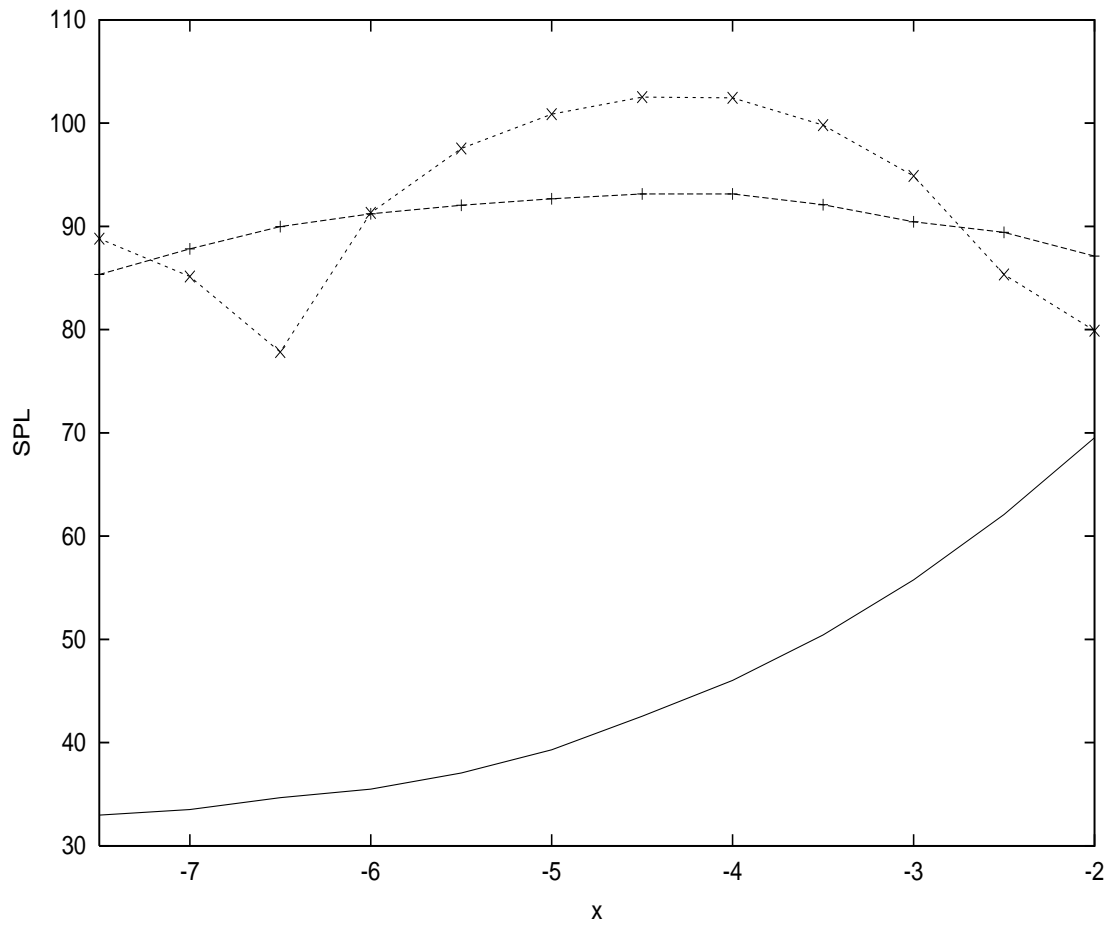

Figure 11: SPL $\left(d B\right.$ re. $\left.2 \times 10^{-5} \mathrm{~N} / \mathrm{m}^{2}\right)$ variation on the line $y=z=0$ for the three configurations: nacelle (full line), fuselage $(+)$, and wing $(\times)$. 


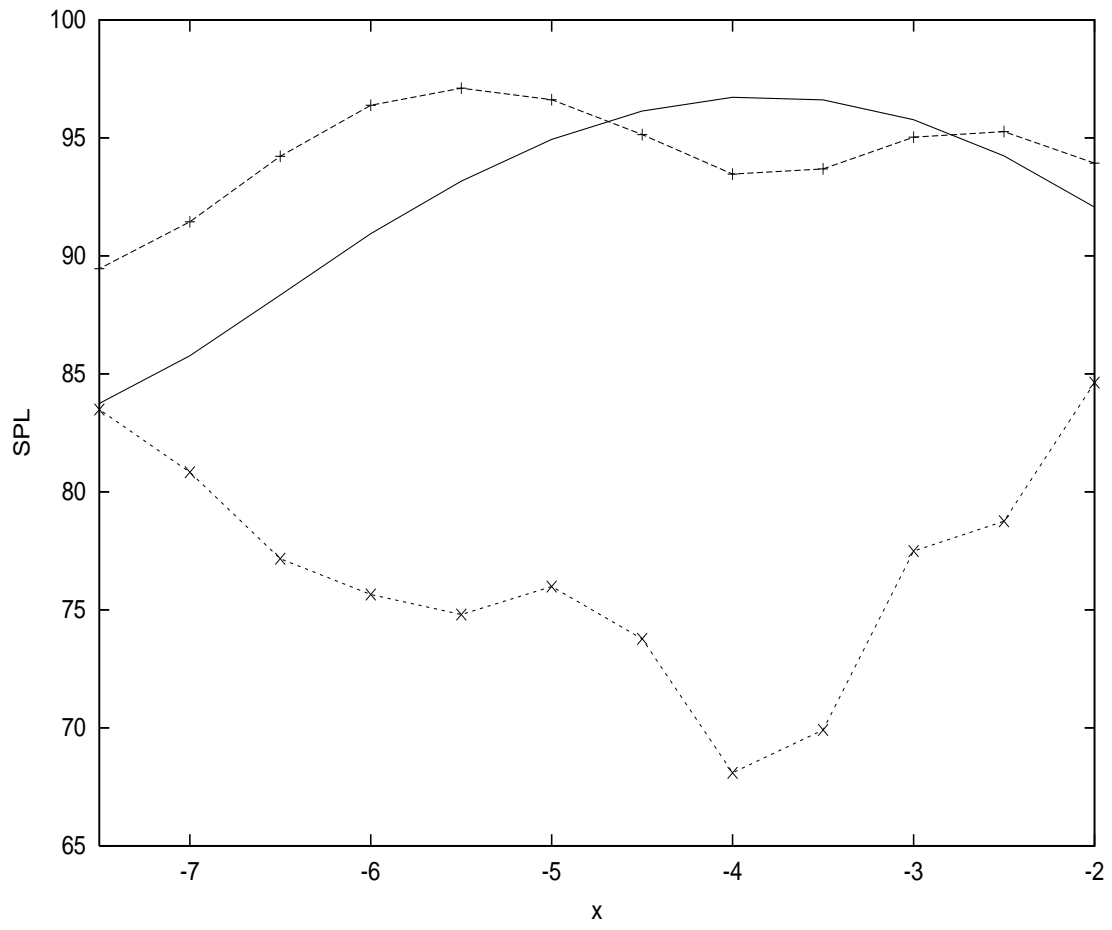

Figure 12: SPL $\left(d B\right.$ re. $\left.2 \times 10^{-5} N / m^{2}\right)$ variation on the line $y=0, z=-4$ for the three configurations: nacelle (full line), fuselage $(+)$, and wing $(\times)$. 